\title{
BMJ Open Implications of involving pharmacy technicians in obtaining a best possible medication history from the perspectives of pharmaceutical, medical and nursing staff: a qualitative study
}

\author{
Andrea Niederhauser, ${ }^{1}$ Chantal Zimmermann, ${ }^{1}$ Liat Fishman, ${ }^{1}$ \\ David L B Schwappach ${ }^{1,2}$
}

To cite: Niederhauser A, Zimmermann C, Fishman L, et al. Implications of involving pharmacy technicians in obtaining a best possible medication history from the perspectives of pharmaceutical, medical and nursing staff: a qualitative study. BMJ Open 2018;8:e020566. doi:10.1136/ bmjopen-2017-020566

- Prepublication history and additional material for this paper are available online. To view these files, please visit the journal online (http://dx.doi org/10.1136/bmjopen-2017020566).

Received 9 November 2017 Revised 9 April 2018 Accepted 20 April 2018
Check for updates

${ }^{1}$ Swiss Patient Safety Foundation, Zürich, Switzerland ${ }^{2}$ Institute of Social and Preventive Medicine (ISPM) University of Bern, Bern, Switzerland

Correspondence to Andrea Niederhauser; niederhauser@ patientensicherheit.ch

\section{ABSTRACT}

Objectives In recent years, the involvement of pharmacy technicians in medication reconciliation has increasingly been investigated. The aim of this study was to assess the implications on professional roles and collaboration when a best possible medication history (BPMH) at admission is obtained by pharmacy technicians.

Design Qualitative study with semistructured interviews. Data were analysed using a qualitative content analysis approach.

Setting Internal medicine units in two mid-sized Swiss hospitals.

Participants 21 staff members working at the two sites (6 pharmacy technicians, 2 pharmacists, 6 nurses, 5 physician residents and 2 senior physicians).

Results Pharmacy technicians generally appreciated their new tasks in obtaining a BPMH. However, they also experienced challenges associated with their new role. Interviewees reported unease with direct patient interaction and challenges with integrating the new BPMH tasks into their regular daily duties. We found that pharmacists played a key role in the BPMH process, since they act as coaches for pharmacy technicians, transmit information to the physicians and reconcile preadmission medication lists with admission orders. Physicians stated that they benefitted from the delegation of administrative tasks to pharmacy technicians. Regarding the interprofessional collaboration, we found that pharmacy technicians in the study acted on a preliminary administrative level and did not become part of the larger treatment team. There was no direct interaction between pharmacy technicians and physicians, but rather, the supervising pharmacists acted as intermediaries. Conclusion The tasks assumed by pharmacy technicians need to be clearly defined and fully integrated into existing processes. Engaging pharmacy technicians may generate new patient safety risks and inefficiencies due to process fragmentation. Communication and information flow at the interfaces between professional groups therefore need to be well organised. More research is needed to understand if and under which circumstances such a model can be efficient and contribute to improving medication safety.
Strengths and limitations of this study

- The qualitative study design allowed an in-depth exploration of the implications for different healthcare professionals when introducing a new interprofessional model.

- By interviewing pharmacy technicians, pharmacists, physicians and nurses, we were able to take into account the perceptions of all healthcare professionals affected by the new model.

- Participants were recruited from two sites only. Findings need to be understood in the local context and are only partly transferable to other settings.

- Participants were recruited by local study coordinators, sampling bias cannot be excluded.

\section{INTRODUCTION}

Transitions of patients between the hospital and other healthcare settings are vulnerable times for medication errors and adverse drug events (ADEs). Discrepancies such as inadvertent omissions and duplications of medications and dosing errors are common at hospital admission and discharge. ${ }^{1-6}$ Unintentional medication changes and communication failures at these interfaces are associated with potential harm and high utilisation of healthcare resources such as readmissions. ${ }^{7-9}$ Medication reconciliation is the process of thoroughly and accurately establishing a patient's medication list and using this list to provide correct medications to the patient. ${ }^{10}$ Over the past decade, medication reconciliation has been advocated in more and more countries as an important patient safety strategy for preventing medication discrepancies at transitions of care. ${ }^{11-17}$

While medication reconciliation (or 'med rec', as it is often called) has strong face validity and a large body of evidence points to 
- Use at least two reliable sources (e.g. medication list from the general practitioner, patient's medication list, drug packages). Whenever possible, one of the sources should be a systematic patient interview conducted with a structured guide.

- Record all current medications (incl. OTC, vitamins, herbals, "as needed" etc.).

- Make preadmission medication list accessible to the whole healthcare team by filing it in a central place.

- Obtain the BPMH within 24 hours after admission

- Define roles and responsibilities for obtaining the BPMH and train accordingly.

Figure 1 Quality standards for obtaining a best possible medication history (BPMH). OTC, over-the-counter.

its benefits, ${ }^{18-21}$ many questions still arise as how to best conduct the process in routine care. An important implementation barrier is the resource intensity that is associated with med rec interventions. ${ }^{22}$ Systematic literature reviews have concluded that many successful interventions in medication reconciliation involved pharmacists. ${ }^{18-20}$ However, implementing interventions in which pharmacists conduct med rec for a majority of hospitalised patients is expensive (though this may still be cost-effective on a systems level by reducing drug expenses and costly $\mathrm{ADEs}^{2324}$ ). In recent years, the involvement of pharmacy technicians, supervised by pharmacists, in medication reconciliation has increasingly been investigated, the assumption being that this may offer many of the advantages of pharmacist-based interventions but at a lower cost. Evidence from numerous studies demonstrates that trained pharmacy technicians are able to gather medication histories with similar completeness and accuracy to other healthcare professionals. ${ }^{25}{ }^{26}$ One study found that, compared with non-pharmacy personnel, pharmacy technicians had an absolute risk reduction of $50 \%$ in medication history errors. ${ }^{27} \mathrm{~A}$ three-arm randomised controlled study found that when adding a best possible medication history (BPMH) by either pharmacy technicians or pharmacists to usual care processes, errors in the admission history were reduced by over $80 \%$. There was no difference in the benefits provided by pharmacy technicians versus pharmacists. ${ }^{28}$

For pharmacy technicians, participating in the medication reconciliation process represents a new professional role not included in their initial training. Obtaining a BPMH, for example, includes a systematic interview with the patient and/or his or her carers in order to capture all medications currently being taken (figure 1). To our knowledge, only few studies have explored the views of pharmacy technicians regarding new patient-centred tasks. The studies concluded that pharmacy technicians indicate a desire to take on more clinical or managerial tasks; however, their current training and education may not sufficiently equip them for these new roles. ${ }^{29}{ }^{30}$ With a growing interest to involve pharmacy technicians in medication reconciliation, it is important to understand how pharmacy technicians perceive their role in the process and to understand the consequences for the interprofessional collaboration within the care team. Knowing more about these aspects may have important implications for successfully implementing this novel interprofessional medication reconciliation model. We, thus, conceived this study in the context of a larger multisite quality improvement programme led by the Swiss Patient Safety Foundation (SPS). The aim of the study was to investigate the new role of pharmacy technicians in obtaining a BPMH from the perspectives of pharmaceutical, medical and nursing staff as well as its implications for the interprofessional collaboration.

\section{METHODS \\ Study design}

This qualitative substudy was conducted as part of the Swiss national quality improvement programme progress! Medication Reconciliation which aimed to promote medication reconciliation in acute care hospitals in Switzerland. The progress! programme was designed and led by the SPS. One of the cornerstones of the programme was to test the feasibility of performing the first step of medication reconciliation, namely obtaining a BPMH at admission. Eight hospitals participated in the programme. Each hospital committed itself to defining and testing a new process for obtaining a BPMH on a designated internal medicine 'pilot unit'. The new process had to be based on quality standards as defined by the programme (figure 1).

The hospitals were otherwise free to design workflows, tools and responsibilities adapted to their local conditions. As a result, the roles and responsibilities for obtaining a BPMH varied among the hospitals. In some hospitals, the BPMH was obtained by physician residents, in others, pharmaceutical staff was involved in the process. In two hospitals, pharmacy technicians (supervised by pharmacists) were responsible for obtaining a BPMH. The present substudy focuses on the experiences with this novel model in these two hospitals.

\section{Setting and processes for obtaining a BPMH}

Both hospitals included in the study are mid-sized acute care hospitals located in Switzerland. In each of the hospitals an interdisciplinary project team defined a process to obtain a BPMH on admission (figure 2). The new process was tested on an internal medicine pilot unit for the duration of 1 year (November 2015 to November 2016). In both hospitals, no pharmaceutical personnel was involved in obtaining a medication history prior to the project. Medication histories were obtained by physician residents and sometimes nurses. Pharmacy technicians already working in the hospital pharmacy were trained for the new role. The SPS programme team supplied a training guide, training resources (eg, role-playing exercises and presentations) as well as a patient interview guide. The training sessions were organised and conducted by the local study teams.

\section{Sample}

Twenty-one semistructured interviews with six pharmacy technicians, two pharmacists, six members of the nursing 


\section{Hospital A:}

$\mathrm{BPMH}$ is obtained for all elective patients admitted to the general internal medicine department and for patients admitted between 12 midnight and 5 am from the emergency department to the general internal medicine department (approx. 30 admissions/month). Pharmacy technicians conduct systematic patient interviews using available information sources and record the preadmission medication list on a special form in the electronic patient chart. Physicians receive the list and document further action for each medication (e.g. stop, continue, modify). They then order the admission medications. Subsequently, clinical pharmacists check the admission orders and provide feedback to the physicians on any discrepancies identified.

\section{Hospital B:}

BPMH is obtained for all patients aged 40 to 85 years admitted through the emergency department to one general internal medicine ward (approx. 40-60 admissions/month). At admission, a medication history is obtained by physician residents. To this end, a new electronic form was introduced where information on preadmission medication is recorded. Physician residents then write the admission orders. Once patients are transferred to the ward, pharmacy technicians conduct a structured patient interview to systematically capture all preadmission medications and record this information on a paper form. If necessary, other sources are consulted for additional information. Pharmacists then compare the preadmission medication to the medication orders, check for potential drug-drug interactions,control doses and provide feedback to the physicians. The paper form is filed in the patient record.

Figure 2 Processes defined to obtain the best possible medication history (BPMH).

staff, five physician residents and two senior physicians were conducted in the two hospitals (table 1 ). The interviews took place during a 2-day site visit from the SPS programme team at 6 months after implementation of the new processes.

Participants were purposely sampled to represent members of all professional groups who are affected by the new BPMH process, that is, pharmacy technicians, pharmacists, physicians and nurses. To be eligible for an interview, pharmacy technicians had to be directly involved in obtaining the BPMH. Pharmacists had to have an active part in supervising the pharmacy technicians in the BPMH. Nurses, residents and senior physicians were recruited from the participating pilot units and had to have experienced the new BPMH process for at least 4 weeks. Due to rotations, not all of the interviewed residents in hospital A still worked on the pilot unit at the time of the interview. One senior physician was not working on the pilot unit, but was responsible for instructing the residents on the new BPMH process and was therefore

\begin{tabular}{|c|c|c|c|}
\hline & Hospital A & Hospital B & Total \\
\hline \multicolumn{4}{|l|}{ Profession } \\
\hline Pharmacy technician & 3 & 3 & 6 \\
\hline (Clinical) pharmacist & 1 & 1 & 2 \\
\hline Resident physician & 3 & 2 & 5 \\
\hline Senior physician & 1 & 1 & 2 \\
\hline Nurse & 3 & 3 & 6 \\
\hline \multicolumn{4}{|l|}{ Gender } \\
\hline Male & 1 & 3 & 4 \\
\hline Female & 10 & 7 & 17 \\
\hline
\end{tabular}

selected to be interviewed. Participants were recruited by the local study coordinator. Written informed consent was obtained from all participants prior to the interview.

\section{Data collection}

The interview guide (see online supplementary appendix 1) was developed and iterated by the SPS programme team. The guide contained several thematic sections. Participants were first invited to describe their own role, as well as the role of other healthcare professionals in obtaining a BPMH. They were then asked to evaluate their collaboration with the other healthcare professionals and whether they had noticed any positive or negative changes or implications for their daily practice since implementation of the new process. Respondents were also asked to comment on the advantages and disadvantages of involving pharmacy technicians in obtaining a BPMH. In the last section, which was not included in the present analysis, participants were asked about the perceived benefits of a BPMH on medication safety, the impact on patients and general suggestions for improving implementation of the new processes. For each section, various subquestions and prompts were included.

The personal interviews were conducted by LF (physician, project lead for the progress! programme) and AN (social scientist). CZ (nurse and social scientist) was present in three of the interviews to take notes. Both interviewers trained in interviewing techniques beforehand. The interviews were conducted face to face at the respective hospital and lasted between 30 and 40 min each. With the exception of the participant and the researchers, no one else was present during the interview. The interviewers introduced themselves and reiterated the background and aims of the study before the interview. Handwritten notes were taken during the interview. The interviews were audio taped and transcribed verbatim by a professional transcriptionist. No repeat interviews were conducted. Transcripts were not returned to participants for comment.

\section{Data analysis}

All analyses were performed by using the software ATLAS.ti V.7. A conventional qualitative content analysis approach was applied. ${ }^{31}{ }^{32}$ The data were analysed by AN and CZ. In a first step, both researchers each read three randomly selected transcripts. They first applied an inductive approach of data coding, taking notes on recurring topics or concepts that emerged from the interviews. Based on this, both researchers developed a first set of codes. These two sets were then compared and merged into one coding structure. The code structure was completed by comparing it to the interview guide. In a next step, both researchers independently applied the new code structure to three more transcripts. The code structure was then discussed and revised anew, as new codes were added and some were removed. This iterative process continued until each of the researchers had coded one half of the transcripts. The code structure 
was reflected, discussed and finalised together with DLBS (senior researcher) and transcripts were recoded accordingly. To increase reliability, AN and CZ jointly applied the finalised code structure to the three initial transcripts. Following this, each of the researchers independently coded the second half of the transcripts. The final coding tree consisted of the following main categories: 'tasks' (in BPMH process), 'impact on own work', 'interprofessional collaboration', 'evaluation of the pharmacy technician's involvement' and 'evaluation of current processes'. Subcategories were developed for a more differentiated organisation of the data content. Through code by code comparison, discrepancies were identified, reviewed together and resolved. These intensive discussions also allowed the two coders to reflect on the personal assumptions and preconceptions that they may have applied to the interviews. Data were organised into major themes relevant to the research question and quotes were analysed accordingly. Final results were discussed in a joint session with all four authors. Findings were not discussed with participants. Quotations were translated from German to English for this publication by the authors.

\section{Patient and public involvement}

Patients or public were not involved in any stages of this study.

\section{RESULTS}

Two major themes emerged to be pertinent to the research question: the impact of the pharmacy technicians' new role on the daily work of all involved healthcare professionals and its impact on the interprofessional collaboration.

\section{Impact on the daily work}

The pharmacy technicians' new role had impacts on the daily work of each professional group. We report the results from the perspective of each of the groups interviewed.

\section{Perspective of pharmacy technicians}

The new BPMH process had positive and negative impacts on the daily work of pharmacy technicians. A majority of them mentioned that their new tasks were interesting or enriching and that they made their day-to-day operations more varied.

For us there is a lot of variety and I like to interact with people, so in this regard I also find it adds value to me and to my profession. Pharmacy technician 4

By conducting structured patient interviews, pharmacy technicians work with patients in a much closer way than they did before. Throughout the interviews it became clear that, unlike nurses or physicians, pharmacy technicians are not much used to, nor specifically trained for direct patient interaction. Most participants reported enjoying the new contact with patients, however, they also mentioned that it had required them to learn new ways on how to interact with patients (eg, using disinfectant before greeting the patient) and how to handle difficult or unexpected situations (eg, when patients are unwilling or unable to answer questions).

You always have to be very careful. Some try to tell you about their entire life. How do you interrupt, without insulting the patient, but so that you know enough? This is difficult at times. Pharmacy technician 4

In hospital A, where pharmacy technicians obtained the BPMH from elective as well as emergency patients, the elective patients were interviewed in a separate booth in the main lobby after registering in the hospital and before being admitted to the ward, whereas emergency patients were interviewed in their ward rooms. The pharmacy technicians in this hospital enjoyed interviewing patients entering for elective treatments, but did not feel at ease interviewing patients on the ward.

I don't find it interesting to go to the ward and into the room. I don't like that too much. Pharmacy technician 3

One pharmacy technician explained that she chose her profession in order to keep a certain distance to the clients. She did not consider herself to be sufficiently trained to adequately handle difficult situations.

And I feel, for me, as a pharmacy technician you enjoy the contact with clients, but not if it's too close. And in the patient room it is different, compared to when the patient is here [in the admission booth]. Here you still have a certain distance. And yes, sometimes I find it difficult upstairs [on the ward], well, depending on the patient you have, because we weren't trained or prepared for something like that. [...] If you then really have to interview a patient who's in serious pain $[\ldots]$, then I don't feel at ease with him. Pharmacy technician 2

Pharmacy technicians in hospital B did not mention this issue of being too close to the patients. However, they only interviewed patients on the ward and therefore did not have a similar opportunity to compare the different situations.

Lastly, the new tasks of pharmacy technicians had a significant impact on the planning of their day-to-day operations. The time required for obtaining a BPMH had to be fit in within their other daily duties. In hospital A, this was perceived as burdensome and stressful by some.

It extremely influences and impairs the daily operations. [...] I manage the medicine stock on the intensive care unit. I'm there and then I'm called, 'someone is here', then I have to leave [the ICU] and come here. I cannot plan my daily operations anymore. They are interrupted or are falling short. The medication history requires a lot of time. Pharmacy technician 3 
It is just difficult in our current situation. You have too little resources, you notice, you have to, or you can, or you should do this as well, and as precisely as possible. $[\ldots]$ and then you make some further inquiries and sometimes it really takes a lot of time. Sometimes not, sometimes it does. And I notice, sometimes this becomes a burden. Pharmacy technician 1

In hospital B, pharmacy technicians also mentioned challenges with integrating the BPMH into their daily routine. However, they also mentioned that they have the option of not obtaining a BPMH if there is too much conflict with their other tasks.

When it gets really bad, you have to say, today we can't do it. But otherwise, I don't feel that stressed because of it. It already happened that I had three or four patients to interview. I arrive [on the ward], but then one is being seen by the doctor, the second is having physiotherapy, the third is in an examination. In the end I have four patients and I would have had the time but could not interview anyone. This has happened before. Pharmacy technician 4

\section{Perspective of pharmacists}

Pharmacists in both hospitals reported an increased workload.

It certainly has gotten more laborious. You have to think about it more, that you have to do it $[\ldots]$ and then the next day we have to check everything, this takes a lot of time. Pharmacist 1

Yes, it is additional, something you do as well. Pharmacist 2

As supervisors, pharmacists coach and advise pharmacy technicians before and after the patient interviews, for example, on how to interact with patients or how to better understand the individual medication in relation to the diagnoses, as illustrated by the following quote:

They are experiencing so much through the interaction with the patients that I try to meet them there too, in order to discuss their experiences, to talk about what the patient told them [...]. On the other hand also to give them explanations about why he takes [a medication]. Pharmacist 2

In hospital A, interview partners explained that an additional check of the preadmission medication list by pharmacists was introduced directly after the pharmacy technicians had obtained a BPMH and before sending it to the physicians. Pharmacists control the plausibility and accuracy of the recorded information, for example, by taking into account patients' diagnoses. This check was not intended in the original design of the BPMH process, but was introduced after pharmacists had received feedback from physician residents that in some cases pharmacy technicians had not recognised the relevance of certain information concerning a patient's medication in relation to his diagnoses. Thus, they had not forwarded important information immediately to the physician.

We received one or two feedbacks that the pharmacy technicians didn't record something that was important. Somehow, marcumar ${ }^{\circledR}$, an anticoagulant, had been discontinued some days before and she had written it down in the comments. And for the physician it is essential to know that [the patient] had it. [...] A physician would have noted it at the very top. But a pharmacy technician has her list and her interview guide that she goes through, and she may not be aware for all the medications how important they are and how they match with a diagnosis. [...] We should have noticed it. From there on, we looked over it before it is sent to the physician. Pharmacist 1

Finally, in both hospitals, pharmacists also conduct the final step of medication reconciliation at admission. Once the patient's preadmission medication list is established by pharmacy technicians, pharmacists compare that list with the admission orders and review the medication orders for potential drug-drug interactions.

And [the pharmacists] also looked at it again and gave us feedback on interactions, or that they cannot find a correct diagnosis for this and that medication. Physician resident 1

\section{Perspective of physicians}

The impact of the BPMH obtained by pharmacy technicians on the daily work of physicians was experienced differently by the respondents of the two hospitals. In hospital A, physicians used the preadmission medication list of the pharmacy technicians in order to write their admission orders. Hence, their work built on the BPMH previously obtained by pharmacy technicians. They noted that involving the pharmacy technicians relieved them of certain time-consuming administrative duties.

The administrative burden [of our work] was clearly reduced. Physician resident 1

And now, everything is already documented and a tedious part of our work is already done. Physician resident 2

In addition, physician residents in hospital A stated that the multistaged processes led to a more systematic and thorough assessment of a patient's medication. They also expressed comfort in the thought that there was someone else looking at the patient's medication.

I think, what is good about it, is that I know that one of the pharmacists will have a look at it, that there certainly is a better control of the medication in general. If there are things that need to be changed. This is certainly something that helps. Physician resident 3

In hospital $\mathrm{B}$, at the start of the project, physician residents were trained to obtain a more complete medication history compared with the previous status quo, and to 
document it in a new, more structured electronic form. In this hospital, the BPMH obtained by pharmacy technicians and pharmacists was implemented in complement, rather than in substitution of the medication history obtained by physicians. The physician residents interviewed did not report any noticeable change to their daily work since pharmacy technicians were involved in obtaining a BPMH. Rather, one of them stated that:

We don't rely on the work of pharmacists or pharmacy technicians. We're trying to obtain a best possible medication history ourselves. Physician resident 4

\section{Perspective of nurses}

In both hospitals, nursing staff was only peripherally involved in obtaining a BPMH. However, all nurses reported experiencing positive impacts on their daily work since implementation of the new BPMH process. Since both hospitals implemented various elements at once (eg, new electronic form, new process for hospital discharge), for nursing staff it was not possible to clearly discern whether it was the work of pharmacy technicians or other elements that contributed to this impact. Notwithstanding, they all stated that medication orders had improved in clarity and completeness.

Prior to this, we often experienced that we came in the morning, distributed the pills and then it was said [by the patients] 'this one is missing and that one is missing'. Then we went to ask [(the doctors]: 'Yes, that's not important.' Or: 'Oh, I didn't know that.' Only those from the general practitioner [were recorded], but something that [the patient] took himself wasn't recorded. Nurse 5

With preadmission medication recorded more thoroughly, the nurses found that they spent less time asking physicians about incomplete or imprecise orders as well as discussing missing medication with patients. Furthermore, one nurse pointed out that knowing that someone else would record all preadmission medication relieved nursing staff from the perceived responsibility of ensuring that the patient received all his necessary medication.

I think it is really a relief for us. Even when patients come in late in the evening or during the night, you don't have to think too much about their medications, rather you know someone will come who conducts an interview with them and records everything. Nurse 4

\section{Impact on interprofessional collaboration}

We were particularly interested in analysing how the involvement of pharmacy technicians would affect the existing structures and team dynamics between the professional groups. The following central themes emerged from the interviews.
Pharmacy technicians act outside of the care team

With the new BPMH process, pharmacy technicians became an additional professional group that requires access to the patient. In spite of this, the interviews suggest that the pharmacy technicians did not become part of the larger care team, but rather assume an outside role. Pharmacy technicians reported having to work their way around other healthcare professionals' schedules and spending a lot of time coordinating with nursing staff to find a good time to conduct the interview.

What can often be a little bit of a problem, maybe there's the nutritionist with the patient, then the physiotherapist comes in, and then we come in as well. Then the patient gets annoyed at some point. So you have to watch this a little bit, then I'll go interview the other two [patients] first and maybe only in the afternoon I'll go see this patient. They are less cooperative, when the physiotherapist is there, she doesn't show consideration for me. They certainly also have their schedule. Pharmacy technician 4

Other healthcare workers do not pay a lot of attention to the pharmacy technicians. This is also illustrated by the fact that some physicians were not able to clearly distinguish between pharmacy technicians and pharmacists in the interviews. Some nurses reported that the presence of pharmacy technicians was hardly noticed on the wards.

Well, we don't actually see them that much. They sneak upstairs quickly $[\ldots]$ and then they do it, actually calmly and quietly, well, they rarely come to ask us anything and then they leave again. Nurse 2

The pharmacy technicians are not really part of our team. They come as if they were guests. Senior physician 2

Some pharmacy technicians also described themselves as 'someone from the outside'. They attributed positive characteristics to this role, stating that this way they could act as a link between the patients and the physicians and gather more information from a patient.

I see myself as an intermediary, somehow. I'm not anyone's competitor, but I'm coming from the outside. I can also see this with the patients. They notice 'aha, someone is coming, she's not part of the nursing staff, she's coming from a different place in the hospital.' I notice that sometimes they tell me almost more $[\ldots .$.$] . Pharmacy technician 4$

\section{No direct interaction between pharmacy technicians and physicians}

Pharmacy technicians and physicians reported that there is almost no direct interaction between them. In each hospital, one of the pharmacy technicians said that she considers herself a 'link' or an 'intermediary' between the physician and the patient. However, the interviews suggest that the way the BPMH processes were defined in both hospitals does not allow pharmacy technicians to fulfil 
such a role. Rather, information flows in one direction only and passes the pharmacists as another checkpoint before reaching the physician. Pharmacy technicians do not know what happens to the information collected from the patient after it has been transmitted from the pharmacists to the physicians and they receive little feedback from the physicians on how their work is perceived.

I can't say much about [the role of the physicians], because it's all done by our pharmacists. I just hand them over the sheet. I tell the pharmacist: "[...] 'here and here you will probably have to take a closer look' or 'here I'm not quite sure', but I hardly ever see a physician. Pharmacy technician 4

On the ward, I do not have any contact with the physicians. Pharmacy technician 3

\section{Strengthened collaboration between pharmacists and physicians} Pharmacists and physicians both reported that their collaboration had intensified since the new medication reconciliation processes were introduced. Because they control the work of pharmacy technicians and reconcile preadmission lists with admission orders, pharmacists were also provided with an opportunity to analyse patients' individual medication and provide feedback on interactions. Physicians in both hospitals found this feedback to be helpful for their work. Pharmacists in turn considered it of high value to be more involved in the medication process and to better know and interact with physicians.

[...] it sometimes happens that the residents approach me about something in the hallway or that they ask questions or call me. It is not just because it's interesting for me, but I also think that it's better for medication safety when one is less inhibited to ask someone, because ... If one asks everything and doesn't hesitate to ask because one doesn't want to disturb. Pharmacist 1

\section{DISCUSSION}

Our study found that pharmacy technicians who obtain a BPMH generally enjoy and benefit from their new tasks. Their daily work becomes more varied and interviewing elective patients in particular seems to be a welcoming change. However, these tasks also come with challenges for pharmacy technicians. Being responsible for conducting the BPMH makes their planning of day-to-day duties more difficult, since the daily number of admissions is not predictable and the pharmacy technicians have to work their way around the schedules of other healthcare professionals. Direct patient interaction can be another challenge for pharmacy technicians, since they are usually not used to, nor trained to be in direct contact with seriously ill patients. Simple questions such as whether or not to shake a patient's hand illustrate the dimension of uncertainty for pharmacy technicians.
This finding underlines the importance of a dedicated training programme for pharmacy technicians on how to obtain a BPMH which includes practical elements such as role-playing exercises, as stated in other studies. ${ }^{25} 33$ Our findings suggest that for some pharmacy technicians, direct patient interaction may be too close and that not all pharmacy technicians may be equally willing and apt to deal with these challenges. This is an aspect that should be considered when recruiting pharmacy technicians for these tasks. Choosing pharmacy technicians who demonstrate good interpersonal, communication and problem-solving skills has been identified as one of the best practices when engaging pharmacy technicians in medication reconciliation efforts. ${ }^{25}$ The situation for pharmacy technicians in our study is different when they are interviewing elective patients at admission, who are generally stable and not in urgent need for medical care. In this case, the neutral space of the admission booth may convey a sense of distance and better control of the situation. As for their place within the team, we found that pharmacy technicians did not become members of the treatment team. They have no proper position awarded in the pre-existing spatial and social structures on the ward, but rather act on a preliminary administrative level.

Our findings indicate a benefit for the daily work of nurses as well as for the integration of pharmacists in the medication process. However, due to the complexity of the processes, it is not clear to what extent these implications are linked to the BPMH being obtained by pharmacy technicians, and to what extent they are a result of the other elements introduced as part of the programme.

The findings suggest that involving pharmacy technicians in obtaining a BPMH can also have positive effects on the daily work of physicians. In particular, physician residents benefit from the delegation of administrative tasks to pharmacy technicians (such as collecting additional sources for the medication history). However, we also found that certain tasks were still performed by pharmacy technicians as well as physicians. In line with other research, ${ }^{34}$ our analysis indicates that physicians may be reluctant to hand off tasks to other professional groups, because they consider obtaining a medication history a cornerstone for deciding on the treatment regime. Boockvar et al found that medication reconciliation is challenging for physicians because it competes for their time with other tasks and that they prioritise more urgent care responsibilities. The authors, thus, suggest that efficacy and perceived capability regarding the completion of medication reconciliation might be improved by dividing the task into parts more easily manageable by individual team members. ${ }^{35}$

The results also demonstrate that there was a shift of workload from physician residents and nurses to pharmacy technicians and pharmacists. In both hospitals, pharmacy technicians and pharmacists performed the BPMH tasks in addition to their other daily duties. As a consequence, they had to interrupt other tasks or at times chose not to obtain a BPMH (hospital B). This 
raises safety concerns, since shifting the attention away from an original task and returning back to it later can increase the likelihood of errors. ${ }^{36}$ In hospital A, more pharmaceutical staff was eventually hired to better redistribute some of the workload. This underlines the fact that without adding additional human resources, such a model can hardly be sustainable.

We also found that in both hospitals, engaging pharmacy technicians in obtaining a BPMH was, at the time of the interviews, not possible without the substantial involvement of pharmacists. Pharmacists coached and supported pharmacy technicians and, in one hospital, controlled their work before it was transmitted to the physicians, thus becoming important intermediaries. This implies that the efficiency gained by delegating administrative tasks from physicians to pharmacy technicians may be lost if pharmacists assume a similarly important role in the new process. As other studies have pointed out, once it is established that a pharmacy technician has enough practice to collect an accurate preadmission medication list, removing routine pharmacist verification could improve efficiency in the process. ${ }^{27}$

It is also important to note that the need for the additional check by pharmacists may be due to different expectations as to what is included in the process of obtaining a 'BPMH'. Obtaining a BPMH means to compile an accurate and complete list of all preadmission medications of a patient. It does not include any sort of clinical analysis of the preadmission medication. If pharmacy technicians are to be involved in obtaining a BMPH, this distinction needs to be clear to all healthcare professionals involved, because it clearly delimits which tasks pharmacy technicians can assume and which tasks need to be performed by other healthcare professionals.

Lastly, transferring the task of obtaining a BPMH from physicians to another professional group adds to the fragmentation of the process. In our study, pharmacy technicians did not become part of the wider care team, there was no direct communication between the pharmacy technicians and the physicians, and pharmacists were added to the process as a third group. This raises patient safety concerns, because it creates new interfaces prone to errors and information loss.

In summary, we found that pharmacy technicians are willing and able to take over responsibilities in obtaining a BPMH. However, their engagement can also generate new risks and inefficiencies, especially if new responsibilities are added on top of their other daily duties, if tasks are not clearly delimited or if a pharmacist's active involvement is required to transmit relevant information from the pharmacy technicians to the physicians. These issues need to be addressed in order for this model to really be a safe and cost-effective alternative to more traditional ways for obtaining a BPMH.

\section{Strengths and limitations}

With this study we shed light on the implications of a specific interprofessional model for obtaining a BPMH.
The sampling of medical, pharmaceutical and nursing staff allowed an in-depth exploration of the perception of all healthcare professionals directly affected by such a new model. However, participants were recruited from two hospitals only. Each hospital designed its own process adapted to its local structures and culture. The understanding of the new BPMH process may have varied among the interviewed staff. Furthermore, in each hospital the implementation of the BPMH was accompanied by the introduction of other tools and processes that had a possible impact on the perceptions and answers of the participants. Results, therefore, need to be interpreted in the local context and findings may not be generalisable to other institutions. Transferability of our findings to other settings and countries may also be limited, because prerequisites and traditional education for pharmacy technicians are likely to vary. Regulatory and cultural factors may determine both choice and design of an intervention that aims to involve pharmacy technicians in medication reconciliation, and how successfully such an intervention can be implemented. Nevertheless, because the main themes emerged across both hospitals and align with the results from other studies, we believe that our findings can be relevant to other hospitals that consider implementing such a model. Since the new process was tested in only two hospital pilot units, the number of staff members eligible to participate was very limited. Hence, it is possible that more themes would have emerged if more staff members could have been interviewed. Staff members were selected for interviews by local study coordinators. We have no knowledge of how many staff members declined to participate. Due to the small number of staff eligible for interview, it is unlikely that many refused. Yet, we cannot exclude the possibility that highly motivated individuals were more likely to be selected for the interviews than colleagues with less interest in the new process. In this case, the interviewees in our sample may have conveyed a more positive view about the effects of the new BPMH process on their roles and daily work. Finally, it is likely that such a new interprofessional model requires some time in order to become established and efficient in the daily routine. Follow-up interviews could have shed more light on the long-term effects of the new model.

\section{CONCLUSION}

To our knowledge, this is the first qualitative study that specifically explored the implications on professional roles and collaboration of transferring certain tasks of obtaining a BPMH to pharmacy technicians. We derive some important implications for practice. First, it is crucial that pharmacy technicians receive sufficient time and training to perform their new tasks. Furthermore, the scope of the tasks assumed by pharmacy technicians needs to be clearly defined and fully integrated into existing processes, so that work is not duplicated. Pharmacy technicians can be involved in establishing a 
complete list of preadmission medication, however, the expertise of pharmacists or physicians is required to analyse potential clinical problems regarding the patient's medication. Ultimately, it remains the physician's responsibility to assess whether treatment is appropriate. Due to a fragmentation of the process, it is essential that communication and information flow at the interfaces between professional groups is well organised. Institutions considering this model need to account for sufficient resources for training and task completion and be attentive to the implications which the involvement of a further professional group has on teamwork dynamics and workflow design. More research is needed to understand if and under which circumstances such a model can be efficient and contribute to higher medication safety.

Acknowledgements The authors would like to thank all participating pharmacy technicians, pharmacists, nurses, physician residents and senior physicians for their willingness to share their thoughts in these interviews. We greatly appreciate the support of the local project teams in organising the interviews in the two hospitals.

Contributors AN, CZ, LF and DLBS participated in the conception and design of this study. AN and LF conducted the interviews. AN, CZ and DLBS analysed the data and interpreted the results. AN, CZ and LF drafted the article. DLBS performed critical review of the article. All authors approved the submission of this article.

Funding This work was supported by the Swiss Federal Office of Public Health (no. 12.003298).

Competing interests None declared.

Patient consent Not required.

Ethics approval This study was conducted as part of a quality improvement programme. The quality improvement programme and all data collected obtained a declaration of no objection from the Ethics Committee of the Canton of Zurich.

Provenance and peer review Not commissioned; externally peer reviewed. Data sharing statement No additional data are available.

Open Access This is an Open Access article distributed in accordance with the Creative Commons Attribution Non Commercial (CC BY-NC 4.0) license, which permits others to distribute, remix, adapt, build upon this work non-commercially, and license their derivative works on different terms, provided the original work is properly cited and the use is non-commercial. See: http://creativecommons.org/ licenses/by-nc/4.0/

(C) Article author(s) (or their employer(s) unless otherwise stated in the text of the article) 2018. All rights reserved. No commercial use is permitted unless otherwise expressly granted.

\section{REFERENCES}

1. Pippins JR, Gandhi TK, Hamann C, et al. Classifying and predicting errors of inpatient medication reconciliation. J Gen Intern Med 2008;23:1414-22.

2. von Klüchtzner W, Grandt D. Influence of hospitalization on prescribing safety across the continuum of care: an exploratory study. BMC Health Serv Res 2015;15.

3. Wong JD, Bajcar JM, Wong GG, et al. Medication reconciliation at hospital discharge: evaluating discrepancies. Ann Pharmacother 2008;42:1373-9.

4. Hellström LM, Bondesson Å, Höglund P, et al. Errors in medication history at hospital admission: prevalence and predicting factors. BMC Clin Pharmacol 2012;12:9.

5. Azzi M, Constantino M, Pont L, et al. Medication safety: an audit of medication discrepancies in transferring type 2 diabetes mellitus (T2DM) patients from Australian primary care to tertiary ambulatory care. Int J Qual Health Care 2014;26:397-403.

6. González-García L, Salmerón-García A, García-Lirola M, et al. Medication reconciliation at admission to surgical departments. $J$ Eval Clin Pract 2016;22:20-5.
7. Cornish PL, Knowles SR, Marchesano R, et al. Unintended medication discrepancies at the time of hospital admission. Arch Intern Med 2005;165:424-9.

8. Forster AJ, Murff HJ, Peterson JF, et al. Adverse drug events occurring following hospital discharge. J Gen Intern Med 2005;20:317-23.

9. Witherington EM, Pirzada OM, Avery AJ. Communication gaps and readmissions to hospital for patients aged 75 years and older: observational study. Qual Saf Health Care 2008;17:71-5.

10. Society of Hospital Medicine. MARQUIS Implementation Manual. A guide for medication reconciliation quality improvement. 2014 http://www.hospitalmedicine.org/Web/Quality_Innovation/ Implementation_Toolkit/MARQUIS/Download_Manua_Medication_ Reconciliation.aspx (cited 13 Sep 2017).

11. Dufay É, Doerper S, Michel B, et al. High 5 s initiative: implementation of medication reconciliation in France a 5 years experimentation. Safety in Health 2017;3.

12. Claeys $\mathrm{C}$, Foulon $\mathrm{V}$, de Winter $\mathrm{S}$, et al. Initiatives promoting seamless care in medication management: an international review of the grey literature. Int J Clin Pharm 2013;35:1040-52.

13. National Institute for Health and Care Excellence. Medicines optimisation: the safe and effective use of medicines to enable the best possible outcomes, NICE Guidelines. 2015 www.nice.org.uk/ guidance/ng5 (cited 29 Aug 2017).

14. The Joint Commission. National patient safety goals effective January 2017. www.jointcommission.org/assets/1/6/NPSG_Chapter HAP_Jan2017.pdf (cited 29 Aug 2017).

15. Accreditation Canada, the Canadian Institute for Health Information, the Canadian Patient Safety Institute, the Institute for Safe Medication Practices Canada. Medication Reconciliation in Canada: Raising The Bar - Progress to date and the course ahead. 2012 https://accreditation.ca/sites/default/files/med-rec-en.pdf (cited 29 Aug 2017).

16. Fishman L, Gehring K, Zimmermann C, et al. Medication reconciliation in the acute care hospital [Der systematische Medikationsabgleich im Akutspital]. Zürich: Stiftung für Patientensicherheit 2015.

17. Australian Commission on Safety and Quality in Health Care. Medication reconciliation. 2017 https://www.safetyandquality.gov.au/ our-work/medication-safety/medication-reconciliation/ (cited 13 Sep 2017).

18. Mekonnen AB, McLachlan AJ, Brien JA. Effectiveness of pharmacistled medication reconciliation programmes on clinical outcomes at hospital transitions: a systematic review and meta-analysis. BMJ Open 2016;6:e010003.

19. Mueller SK, Sponsler KC, Kripalani S, et al. Hospital-based medication reconciliation practices: a systematic review. Arch Intern Med 2012;172:1057-69.

20. Kwan JL, Lo L, Sampson M, et al. Medication reconciliation during transitions of care as a patient safety strategy: a systematic review. Ann Intern Med 2013;158(5 Pt 2):397-403.

21. De Oliveira GS, Castro-Alves LJ, Kendall MC, et al. Effectiveness of pharmacist intervention to reduce medication errors and health-care resources utilization after transitions of care: a meta-analysis of randomized controlled trials. J Patient Saf 2017.

22. Pevnick JM, Shane R, Schnipper JL. The problem with medication reconciliation. BMJ Qual Saf 2016;25:726-30.

23. Etchells $\mathrm{E}, \mathrm{Koo} \mathrm{M}$, Daneman N, et al. Comparative economic analyses of patient safety improvement strategies in acute care: a systematic review. BMJ Qual Saf 2012;21:448-56.

24. Najafzadeh M, Schnipper JL, Shrank WH, et al. Economic value of pharmacist-led medication reconciliation for reducing medication errors after hospital discharge. Am J Manag Care 2016;22:654-61.

25. Irwin AN, Ham Y, Gerrity TM. Expanded roles for pharmacy technicians in the medication reconciliation process: a qualitative review. Hosp Pharm 2017;52:44-53.

26. Henriksen JP, Noerregaard S, Buck TC, et al. Medication histories by pharmacy technicians and physicians in an emergency department. Int J Clin Pharm 2015;37:1121-7.

27. Rubin EC, Pisupati R, Nerenberg SF. Utilization of pharmacy technicians to increase the accuracy of patient medication histories obtained in the emergency department. Hosp Pharm 2016;51:396-404.

28. Pevnick JM, Nguyen C, Jackevicius CA, et al. Improving admission medication reconciliation with pharmacists or pharmacy technicians in the emergency department: a randomised controlled trial. BMJ Qual Saf 2017:bmjqs-2017-006761.

29. Boughen M, Sutton J, Fenn T, et al. Defining the role of the pharmacy technician and identifying their future role in medicines optimisation. Pharmacy 2017;5:40. 
30. Maidment ID, Hallows D, Macfarlane H. Does the design of the current pre-registration and post-registration training adequately prepare pharmacy technicians for their current clinical role within mental health? Int J Pharm Pract 2017;25(Suppl 1):40-65.

31. Mayring P. Qualitative content analysis. 2009 http://www.qualitativeresearch.net/fqs-texte/2-00/2-00mayring-e.htm (cited 8 Jul 2013).

32. Hsieh HF, Shannon SE. Three approaches to qualitative content analysis. Qual Health Res 2005;15:1277-88.

33. Cooper JB, Lilliston M, Brooks D, et al. Experience with a pharmacy technician medication history program. Am J Health Syst Pharm 2014;71:1567-74.
34. van Sluisveld N, Zegers M, Natsch S, et al. Medication reconciliation at hospital admission and discharge: insufficient knowledge, unclear task reallocation and lack of collaboration as major barriers to medication safety. BMC Health Serv Res 2012;12:170.

35. Boockvar KS, Santos SL, Kushniruk A, et al. Medication reconciliation: barriers and facilitators from the perspectives of resident physicians and pharmacists. J Hosp Med 2011;6:329-37.

36. Rivera-Rodriguez AJ, Karsh BT. Interruptions and distractions in healthcare: review and reappraisal. Qual Saf Health Care 2010;19:304-12. 